\title{
EXISTENCE OF MOMENTS OF INCREASING PREDICTABLE PROCESSES ASSOCIATED WITH ONE- AND TWO-PARAMETER POTENTIALS
}

\author{
YU. MISHURA and YA. OLTSIK \\ Kiev University \\ Department of Mathematics \\ Kiev, Ukraine 252601
}

(Received March, 1997; Revised May, 1998)

\begin{abstract}
The criterion and sufficient condition for the existence of moments of oneparameter increasing predictable processes is presented in terms of an associated potential. The estimates of moments of special functional connected with two-parameter increasing predictable processes are given in the case when the associated potential is bounded. The application of these estimates to the local time for purely discontinuous strong martingales in the plane is also presented.
\end{abstract}

Key words: Increasing Predictable Process, Associated Potential, Moments of all Orders, Local Time, Discontinuous Strong Martingale.

AMS subject classifications: $60 \mathrm{~J} 45,60 \mathrm{G} 60,60 \mathrm{G} 48,60 \mathrm{~J} 55$.

\section{Introduction, Definitions and Notations}

We estimate the moments of one- and two-parameter increasing processes in terms of associated potentials. It is well known that a one-parameter increasing predictable process has moments of all orders if the associated potential is bounded. In Section 2 we give the criterion and sufficient conditions for the existence of moments of all orders for an increasing process in the case when the associated potential is unbounded, with examples that demonstrate the optimality of the criterion. In Section 3 we give estimates of moments for special functionals connected with increasing predictable two-parameter processes in the case where the associated potential is bounded. The problem of estimating moments of increasing predictable processes associated with two-parameter potentials arises from a question whether the local time for two-parameter purely discontinuous strong martingales has all finite moments. Note that in a one-parameter case Bass [1] gave a positive answer for this question. In Section 4 we apply the results of Section 3 to the local time for purely discontinuous strong martingales on the plane. We estimate moments of special functionals connected with the local time, and formulate the conditions sufficient for the local time to have all finite moments. 
The following are necessary definitions and notations: For two points $s=\left(s_{1}, s_{2}\right)$ and $t=\left(t_{1}, t_{2}\right)$ in $R_{+}^{2}, s \leq t$ means that $s_{1} \leq t_{1}$ and $s_{2} \leq t_{2}$, and $s<t$ means that $s_{1}<t_{1}$ and $s_{2}<t_{2}$. If $s<t$, then $(s, t]$ is the rectangle $\left(s_{1}, t_{1}\right] \times\left(s_{2}, t_{2}\right]$. Let $(\Omega, \mathscr{F}, \mathcal{P})$ be a complete probability space with the filtration $\left\{\mathscr{F}_{t}, t \in R_{+}^{2}\right\}$ which satisfies the following properties [2]:

(F1) if $t<t^{\prime}$ then $\mathscr{F}_{t} \subset \mathscr{F}_{t^{\prime}}$;

(F2) $\mathcal{F}_{0}$ contains all null sets of $\mathcal{F}$;

(F3) for each $t, \mathcal{F}_{t}=\bigcup_{t<t^{\prime}} \mathscr{F}_{t^{\prime}}$;

(F4) for each $t, \mathcal{F}_{t}^{1}$ and $\mathcal{F}_{t}^{2}$ are conditionally independent given $\mathcal{F}_{t}$,

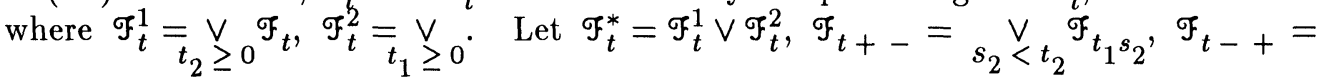
$\underset{s_{1}}{\vee}<t_{1} \mathcal{F}_{s_{1} t_{2}}, \mathscr{F}_{t-}=\underset{s<t}{\vee} \mathcal{F}_{s}, \mathscr{F}_{t-}^{1}=\underset{s_{1}<t_{1}, t_{2} \geq 0}{\vee} \mathcal{F}_{s_{1}}, t_{2}, \mathscr{F}_{t-s_{s_{2}}^{2}<t_{2}, t_{1} \geq 0}^{\vee} \mathscr{F}_{t_{1} s_{2}}$. The definitions of strong, weak 1- and 2-martingale, and increasing process in the plane will follow those of [2]. The definitions and notation of two-parameter predictable, 1and 2-predictable processes, predictable and dual predictable projection will follow those of [7]. The definition of one-parameter potential will follow that of [3]. If $[0, t] \subset R_{+}^{2}$, we denote $\lambda_{i}^{n}=\left\{0=t_{i}^{0}<t_{i}^{1}<\cdots<t_{i}^{n}=t_{i}\right\}, \quad i=1,2, \quad \lambda_{t}^{n}=\lambda_{1}^{n} \times \lambda_{2}^{n}$, $\lambda_{t}^{m} \supset \lambda_{t}^{n}$ if $m>n$, the partition of $[0, t]$. We also denote $t_{i k}=\left(t_{1}^{i}, t_{2}^{k}\right), X_{i k}=X_{t_{i k}}$, $\square_{i k}=\left(t_{i k}, t_{i+1 k+1}\right], \quad \Delta_{i k}^{1} X=X_{i+1 k}-X_{i k}, \quad \Delta_{i k}^{2} X=X_{i k+1}-X_{i k}, \quad \square_{i k} X=$ $\Delta_{i k+1}^{1} X-\Delta_{i k}^{1} X$. The increment of process $X$ on the rectangle $(s, t]$ is $X(s, t]=X_{t}-X_{s_{1} t_{2}}-X_{t_{1} s_{2}}+X_{s}$. Let $\Delta^{1} X_{t}=X_{t}-X_{t-+}, \Delta^{2} X_{t}=X_{t}-X_{t+-}$, $\square X_{t}=X_{t}-X_{t-+}-X_{t+-}+X_{t-}, \quad$ where $X_{t+}=\lim _{s \rightarrow t, s \in Q_{t}^{1}} X_{s}, \quad X_{t-}=\lim _{s \rightarrow t, s \in Q_{t}^{3}} X_{s}$,

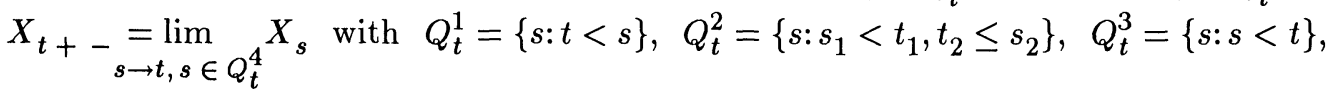
$Q_{t}^{4}=\left\{s: t_{1} \leq s_{1}, s_{2}<t_{2}\right\}$. All processes are assumed to have these limits, to be constant on $\Gamma=(\{0\} \times[0, \infty)) \cup([0, \infty) \times\{0\})$, and to be continuous in $Q_{t}^{1}$.

A functional

$$
\int_{[0, t]} X_{t} d Y_{t}=P-\lim _{\left|\lambda_{t}^{n}\right| \rightarrow 0} \sum_{i, k=0}^{n-1} X_{i k} \square_{i k} Y
$$

is called a stochastic integral of the first kind if this limit exists for any sequence of partitions $\lambda_{t}^{n}$. A functional

$$
\int_{[0, t]} X_{t} d_{1} Y_{t} d_{2} Z_{t}=P \underset{\left|\lambda_{t}^{n}\right| \rightarrow 0}{ } \sum_{i, k=0}^{n-1} X_{i k} \Delta_{i k}^{1} Y \Delta_{i k}^{2} Z
$$

is called a stochastic integral of the second kind if this limit exists for any sequence of partitions $\lambda_{t}^{n}$.

A process $X=\left\{X_{t}, t \in R_{+}^{2}\right\}$ is called a weak submartingale if $X_{t}$ is integrable for each $t, X$ is adapted and $E\left(X(s, t] / \mathscr{F}_{s}\right) \geq 0$ for each $s \leq t$.

If $\left\{A_{t}, t \in R_{+}^{2}\right\}$ is an increasing process, then define $A_{t_{1} \infty}=\lim _{t_{2} \rightarrow \infty} A_{t}, A_{\infty t_{2}}=$ $\lim _{t_{1} \rightarrow \infty} A_{t}, A_{\infty}=\lim _{t \rightarrow \infty} A_{t}$. 
A process $\left\{X_{t}, t \in R_{+}^{2}\right\}$ is called a two-parameter potential if $X_{t}$ is a nonnegative weak submartingale such that $X_{t_{1}}\left(t_{2}\right)$ and $X_{t_{2}}\left(t_{1}\right)$ are one-parameter potentials. If a potential $X_{t}$ is bounded, then it is associated ${ }^{2}$ with the increasing predictable process $A_{t}$ in the following way [5]

$$
X_{t}=A_{t}+E\left(A_{\infty}-A_{t_{1} \infty}-A_{\infty t_{2}} / \mathcal{F}_{t}\right)
$$

\section{Some Properties of One-Parameter Potentials}

Let $\left\{X_{t}, \mathcal{F}_{t}, t \in R_{+}\right\}$be a one-parameter potential associated with predictable increasing process $A_{t}$. It means that $X_{t}=E\left(A_{\infty}-A_{t} / \mathscr{F}_{t}\right)[3]$. All increasing processes are assumed to be integrable.

Theorem 1: The following statements are equivalent:

(a) for every $k \geq 1, E \sup _{t} X_{t}^{k} \leq c_{k}<\infty$,

(b) for every $k \geq 1, E A_{\infty}^{k} \leq d_{k}<\infty$.

Proof: Suppose condition $(a)$ holds. Let $A_{t}^{(n)}=A_{t} \wedge n, X_{t}^{(n)}$ be a potential associated with $A_{t}^{(n)}$. Then for any $k \geq 2$

$$
\begin{gathered}
E\left[A_{\infty}^{(n)}\right]^{k}=E A_{\infty}^{(n)}\left[A_{\infty}^{(n)}\right]^{k-1}=E \int_{0}^{\infty}\left(X_{t}^{(n)}+A_{t}^{(n)}\right) d\left[A_{t}^{(n)}\right]^{k-1} \\
=E \int_{0}^{\infty}\left(X_{t-}^{(n)}+A_{t-}^{(n)}\right) d\left[A_{t}^{(n)}\right]^{k-1}
\end{gathered}
$$

i.e.,

$$
2 E\left[A_{\infty}^{(n)}\right]^{k}=E \int_{0}^{\infty}\left(X_{t}^{(n)}+X_{t-}^{(n)}+A_{t}^{(n)}+A_{t-}^{(n)}\right) d\left[A_{t}^{(n)}\right]^{k-1}
$$

On the other hand, we have from integration by parts that

$$
\begin{aligned}
& 2 E\left[A_{\infty}^{(n)}\right]^{k}=E \int_{0}^{\infty}\left(A_{t}^{(n)}+A_{t-}^{(n)}\right) d\left[A_{t}^{(n)}\right]^{k-1} \\
& +E \int_{0}^{\infty}\left(\left[A_{t}^{(n)}\right]^{k-1}+\left[A_{t-}^{(n)}\right]^{k-1}\right) d A_{t}^{(n)}
\end{aligned}
$$

hence,

$$
E \int_{0}^{\infty}\left(X_{t}^{(n)}+X_{t-}^{(n)}\right) d\left[A_{t}^{(n)}\right]^{k-1}=E \int_{0}^{\infty}\left(\left[A_{t}^{(n)}\right]^{k-1}+\left[A_{t-}^{(n)}\right]^{k-1}\right) d A_{t}^{(n)}
$$

We can estimate the integral in the left-hand side of (3)

$$
E \int_{0}^{\infty}\left(X_{t}^{(n)}+X_{t-}^{(n)}\right) d\left[A_{t}^{(n)}\right]^{k-1}
$$




$$
\begin{gathered}
=E \int_{0}^{\infty}\left(X_{t}^{(n)}+X_{t-}^{(n)}\right) d\left[A_{t}^{(n)}\right]^{k-1} I\left\{\sup _{t} X_{t}^{(n)}>\frac{A_{\infty}^{(n)}}{4 k}\right\} \\
+E \int_{0}^{\infty}\left(X_{t}^{(n)}+X_{t-}^{(n)}\right) d\left[A_{t}^{(n)}\right]^{k-1} I\left\{\sup _{t} X_{t}^{(n)}<\frac{A_{\infty}^{(n)}}{4 k}\right\} \\
\leq 8 k E\left(\sup _{t} X_{t}^{(n)}\right)^{k}+\frac{1}{2 k} E\left[A_{\infty}^{(n)}\right]^{k} .
\end{gathered}
$$

Note that

$$
\begin{gathered}
\int_{0}^{\infty} A_{t}^{(n)} d\left[A_{t}^{(n)}\right]^{k-1}=\lim _{\left|\lambda_{t}^{n}\right| \rightarrow 0} \sum_{k=0}^{n-1} A_{t_{i}}^{(n)}\left(\left[A_{t_{i}}^{(n)}\right]^{k-1}-\left[A_{t_{i-1}}^{(n)}\right]^{k-1}\right) \\
\leq(k-1) \lim _{\left|\lambda_{t}^{n}\right| \rightarrow 0} \sum_{k=0}^{n-1}\left[A_{t_{i}}^{(n)}\right]^{k-1} \Delta A_{t_{i}}^{(n)}=(k-1) \int_{0}^{\infty}\left[A_{t_{i}}^{(n)}\right]^{k-1} d A_{t}^{(n)} .
\end{gathered}
$$

From (2)-(4) we have

$$
E\left[A_{\infty}^{(n)}\right]^{k} \leq k E \int_{0}^{\infty}\left[A_{t_{i}}^{(n)}\right]^{k-1} d A_{t}^{(n)} \leq 8 k^{2} E\left(\sup _{t} X_{t}^{(n)}\right)^{k}+\frac{1}{2} E\left[A_{\infty}^{(n)}\right]^{k}
$$

or

$$
E\left[A_{\infty}^{(n)}\right]^{k} \leq 16 k^{2} E\left(\sup _{t} X_{t}^{(n)}\right)^{k}
$$

Since $X_{t}^{(n)}=E\left(A_{\infty} \wedge n-A_{t} \wedge n / \mathcal{F}_{t}\right) \leq X_{t}$, we have that

$$
E\left[A_{\infty}^{(n)}\right]^{k} \leq 16 k^{2} E \sup _{t} X_{t}^{k}
$$

While $n \rightarrow \infty$, we obtain

$$
E A_{\infty}^{k} \leq 16 k^{2} E \sup _{t} X_{t}^{k}
$$

Suppose condition $(b)$ holds. Then obviously $X_{t} \leq E\left(A_{\infty} / \mathscr{F}_{t}\right)$, and by Doob's inequality

$$
E \sup _{t} X_{t}^{k} \leq \sup _{t}\left(E\left(A_{\infty} / \mathscr{F}_{t}\right)\right)^{k} \leq\left(\frac{k}{k-1}\right)^{k} E A_{\infty}^{k}
$$

The theorem is proved.

Remark 1: Theorem 1 generalizes the well known result that if an associated potential is bounded, then the increasing process has all bounded moments. We give examples to demonstrate that the existence of moments of $X_{t}$ does not supply the existence of moments of $A_{t}$. This means that the conditions of Theorem 1 are quite optimal.

Example 1: Suppose $\left\{\xi_{n}, n \geq 1\right\}$ is a sequence of independent random variables, and each of them has gamma-distribution with parameters $\alpha_{n}=\frac{1}{n^{2}}$ and $\beta_{n}=\frac{1}{\sqrt{n}}$. Then $E \xi_{n}^{k}=\frac{1}{\beta_{n}^{k}}\left(\alpha_{n}+k-1\right) \cdot \ldots \cdot\left(\alpha_{n}+1\right) \alpha_{n}<\infty$. Consider the filtration $\mathscr{F}_{n}=\sigma\left\{\xi_{k}\right.$, $k \leq n\}$ and an increasing predictable process $A_{n}=\sum_{k=1}^{n-1} \xi_{k}$. Hence, a potential associat- 
ed with $A_{n}$ is

$$
X_{n}=E\left(A_{\infty}-A_{n} / \mathcal{F}_{n}\right)=E \sum_{k=n+1}^{\infty} \xi_{k}+\xi_{n}
$$

Note that $X_{n}$ is unbounded, but it has all bounded moments. It is easy to see that $E A_{\infty}=\sum_{n=1}^{\infty} \frac{1}{n \sqrt{n}}<\infty$, but $E A_{\infty}^{2} \geq \sum_{n=1}^{\infty} \frac{n^{2}+1}{n^{3}}=\infty$.

Let $\alpha_{n}=\frac{1}{n^{2}}$ and $\beta_{n}=1$. One can check that a random variable $\eta=\sum_{n=1}^{\infty} \xi_{n}$ has gamma-distribution with parameters $\alpha=\frac{\pi^{2}}{6}$ and $\beta=1$. Therefore, for any $k \geq 1$ $E A_{\infty}^{k}=\left(\frac{\pi^{2}}{6}+k-1\right) \cdot \ldots \cdot \frac{\pi^{2}}{6}<\infty$, i.e., the increasing process has all bounded moments.

Example 2: Consider a standard Wiener process $\left\{w_{t}, \mathscr{F}_{t}, t \in R_{+}\right\}$. Let $\zeta_{t}=$ $e^{a w_{t}-b t}$, where $a, b \in R, b>0$. Then $E \zeta_{t}^{k}=e^{\left(\frac{k^{2} a^{2}}{2}-k b\right) t}<\infty, t>0, k \geq 1$, i.e., $\zeta_{t}$ has all moments. By Itô's formula

$$
\zeta_{t}^{k}=1+k a \int_{0}^{t} \zeta_{s}^{k} d w_{s}+\left(\frac{k^{2} a^{2}}{2}-k b\right) \int_{0}^{t} \zeta_{s}^{k} d s
$$

If $k \leq \frac{2 b}{a^{2}}$, then $\zeta_{t}^{k}$ is a potential; if $k=\frac{2 b}{a^{2}}$, then $\zeta_{t}^{k}$ is a martingale; and if $k>\frac{2 b}{a^{2}}$, then $\zeta_{t}^{k}$ is a submartingale. Suppose $\frac{2 b}{a^{2}}>1$. Then $\zeta_{t}$ is a potential with $A_{\infty}=$ $\left(-\frac{a^{2}}{2}+b\right) \int_{0}^{\infty} \zeta_{s} d s$. Suppose there exists such $n \in N$ that $2^{n-1}<\frac{b}{a^{2}}<2^{n}$. Consider the following cases:

(i) Let $k=2^{n}$. Then $k<\frac{2 b}{a^{2}}, \zeta_{t}^{i}, i \leq k$ is a potential, $\zeta_{\infty}^{i}=0, i \leq k$, and from (5) (the constants depend on $i$ and $r$ )

$$
E\left(\int_{0}^{\infty} \zeta_{s}^{i} d s\right)^{r} \leq c_{1}+c_{2} E\left(\int_{0}^{\infty} \zeta_{s}^{i} d w_{s}\right)^{r}
$$

for every $r \geq 1$. Moreover, by Burkholder's inequalities

$$
c_{3} E\left(\int_{0}^{\infty} \zeta_{s}^{i} d s\right)^{r} \leq E\left(\int_{0}^{\infty} \zeta_{s}^{i / 2} d w_{s}\right)^{2 r} \leq c_{4} E\left(\int_{0}^{\infty} \zeta_{s}^{i} d s\right)^{r}
$$

From (6) and the second part of (7) we obtain

$$
\begin{gathered}
E\left(\int_{0}^{\infty} \zeta_{s} d s\right)^{k} \leq c_{5}+c_{6} E\left(\int_{0}^{\infty} \zeta_{s} d w_{s}\right)^{k} \leq c_{5}+c_{7} E\left(\int_{0}^{\infty} \zeta_{s}^{2} d s\right)^{k / 2} \leq \ldots \\
\leq c_{8}+c_{9} E\left(\int_{0}^{\infty} \zeta_{s}^{2^{n}} d s\right)=c_{8}+c_{9} E \int_{0}^{\infty} e^{k a w_{s}-k b s} d s
\end{gathered}
$$


Thus, $E A_{\infty}^{k}<\infty$.

$$
=c_{8}+c_{9} \int_{0}^{\infty} e^{\left(\frac{k^{2} a^{2}}{2}-k b\right) s} d s<\infty
$$

(ii) Let $k=2^{n+1}$. Then $\zeta_{t}^{i}, i \geq k$ is a submartingale,

$$
E \zeta_{t}^{i}=e^{\left(\frac{i^{2} a^{2}}{2}-i b\right) t} \rightarrow \infty, t \rightarrow \infty ; E\left(\int_{0}^{\infty} \zeta_{s}^{i} d s\right)=\infty
$$

Hence, from the first part of (7) $E\left(\int_{0}^{\infty} \zeta_{s}^{k / 2} d w_{s}\right)^{2}=\infty$. Further, from the potential property and from (5) $E\left(\int_{0}^{\infty} \zeta_{s}^{k / 2} d s\right)^{2}=\infty$. Again, from the first part of (7) $E\left(\int_{0}^{\infty} \zeta_{s}^{k / 4} d w_{s}\right)^{4}=\infty$, and from $(5) E\left(\int_{0}^{\infty} \zeta_{s}^{k / 4} d s\right)^{4}=\infty$. Continuing in the same way, we obtain that $E\left(\int_{0}^{\infty} \zeta_{s} d s\right)^{k}=\infty$; i.e., $E A_{\infty}^{k}=\infty$.

From $(i)$ and $\left(\right.$ ii) we have that $E A_{\infty}^{i}<\infty$ for $i<2^{n-1}<\frac{b}{a^{2}}$ and $E A_{\infty}^{i}=\infty$ for $i \geq 2^{n} \geq \frac{b}{a^{2}}$.

Now we will prove a sufficient condition for the existence of moments $E A_{\infty}^{k}, k \geq 1$ in the case when a potential $X_{t}=E\left(A_{\infty}-A_{t} / \mathscr{F}_{t}\right)$ and associated increasing process $A_{t}$ are continuous. Denote a martingale $M_{t}=E\left(A_{\infty} / \mathscr{F}_{t}\right),\langle M\rangle_{t}$ is its quadratic characteristic.

Theorem 2: Let $X_{t}$ be a continuous one-parameter potential, associated with continuous increasing process $A_{t}$. Assume that for every $k \geq 1, E \int_{0}^{\infty} X_{s}^{k} d\langle M\rangle_{s}<\infty$.
Then

$$
E A_{\infty}^{k}<\infty, \quad k \geq 1
$$

Proof: By Itô's formula for every $k \geq 2$

Therefore,

$$
X_{t}^{k}=k \int_{0}^{t} X_{s}^{k-1} d X_{s}+\frac{k(k-1)}{2} \int_{0}^{t} X_{s}^{k-2} d\langle M\rangle_{s}
$$

$$
\sup _{t} X_{t}^{k} \leq k \sup _{t}\left|\int_{0}^{t} X_{s}^{k-1} d M_{s}\right|+\frac{k(k-1)}{2} \int_{0}^{\infty} X_{s}^{k-2} d\langle M\rangle_{s} .
$$

If the condition of the theorem holds, then by Gundy's inequality

$$
E \sup _{t} X_{t}^{k} \leq k E\left(\int_{0}^{\infty} X_{s}^{2 k-2} d\langle M\rangle_{s}\right)^{1 / 2}+\frac{k(k-1)}{2} E \int_{0}^{\infty} X_{s}^{k-2} d\langle M\rangle_{s}<\infty
$$

hence, by Theorem 1 we have the proof.

Remark 2: Suppose $\langle M\rangle_{t}=\int_{0}^{t} a_{s}^{2} d s$, where $a_{s}$ is a nonrandom function with $\int_{0}^{\infty} a_{s}^{2} d s<\infty$. Then $E \int_{0}^{\infty} X_{s}^{k} d\langle M\rangle_{s}=\int_{0}^{\infty} E X_{s}^{k} a_{s}^{2} d s$. Since $E X_{s}^{k} \rightarrow 0$ when $s \rightarrow \infty$, then a 
continuous function $E X_{s}^{k}$ is bounded and, therefore, $E \int_{0}^{\infty} X_{s}^{k} d\langle M\rangle_{s}<\infty$.

\section{Two-Parameter Potentials Associated with Increasing Processes}

We assume in this section that $\left\{X_{t}, \sigma_{t}, t \in R_{+}^{2}\right\}$ is a two-parameter, nonnegative, bounded potential associated with increasing predictable integrable process $A_{t}$; i.e., (1) holds.

Theorem 3: If $X_{t} \leq c$, then for every $p \geq 1$

$$
E\left(E\left(A_{\infty}-A_{t} / \mathscr{F}_{t}\right)\right)^{p} \leq 2^{p+1} c^{p} p !
$$

Proof: Fix $t_{2} \geq 0$. Consider a process

$$
Z_{t_{1}}\left(t_{2}\right)=E\left(A_{t_{1} \infty}-A_{t} / \mathscr{F}_{t}\right), t_{1} \geq 0
$$

It is obvious that $Z_{t_{1}}\left(t_{2}\right)$ is 1-predictable with respect to the filtration $\left\{\mathscr{F}_{t}^{1}, t_{1} \geq 0\right\}$. Since $A_{t_{1} \infty}-A_{t}$ is $F_{t}^{1}$-measurable and from the condition (F4) [2] we have

$$
Z_{t_{1}}\left(t_{2}\right)=E\left(E\left(A_{t_{1} \infty}-A_{t} / \mathcal{F}_{t}^{1}\right) / \mathcal{F}_{t}^{2}\right)=E\left(A_{t_{1} \infty}-A_{t} / \mathcal{F}_{t}^{2}\right)
$$

Therefore, $Z_{t_{1}}\left(t_{2}\right)$ is predictable in $t_{1}$. Moreover, if $s_{1}<t_{1}$, then

$$
Z_{t_{1}}\left(t_{2}\right)-Z_{s_{1}}\left(t_{2}\right)=E\left(A_{t_{1} \infty}-A_{t}-A_{s_{1} \infty}+A_{s_{1} t_{2}} / \mathscr{F}_{t}^{2}\right) \geq 0
$$

Thus, $Z_{t_{1}}\left(t_{2}\right)$ is a predictable increasing integrable process as a one-parameter process with a parameter $t_{1}$. Denote $X_{t_{1}}\left(t_{2}\right)$ a potential associated with $Z_{t_{1}}\left(t_{2}\right)$. Then from (8)

$$
\begin{gathered}
X_{t_{1}}\left(t_{2}\right)=E\left(Z_{\infty}\left(t_{2}\right)-Z_{t_{1}}\left(t_{2}\right) / \mathcal{F}_{t}\right) \\
=E\left(A_{\infty}-A_{\infty t_{2}}-A_{t_{1} \infty}+A_{t} / \mathscr{F}_{t}\right)=X_{t} .
\end{gathered}
$$

Consequently, $X_{t_{1}}\left(t_{2}\right)$ is bounded; and we can apply the Garsia inequality [3] that leads to the estimate $E\left(Z_{\infty}\left(t_{2}\right)\right)^{p} \leq c^{p} p$ ! or

$$
E\left(E\left(A_{\infty}-A_{\infty t_{2}} / \mathscr{F}_{t}^{2}\right)\right)^{p} \leq c^{p} p !, \quad p \geq 1 .
$$

It follows easily from the last inequality and from (F4) [2] that for every $t \in R_{+}^{2}$

$$
E\left(E\left(A_{t_{1} \infty}-A_{t} / \mathcal{F}_{t}\right)\right)^{p} \leq c^{p} p !
$$

and by a symmetric argument

$$
E\left(E\left(A_{\infty t_{2}}-A_{t} / \mathscr{F}_{t}\right)\right)^{p} \leq c^{p} p !
$$

Finally, we obtain

$$
E\left(E\left(A_{\infty}-A_{t} / \mathcal{F}_{t}\right)\right)^{p} \leq 2^{p} E\left(E\left(A_{\infty}-A_{\infty t_{2}} / \mathscr{F}_{t}\right)\right)^{p}
$$




$$
+2^{p} E\left(E\left(A_{\infty t_{2}}-A_{t} / \mathscr{F}_{t}\right)\right)^{p} \leq 2^{p+1} c^{p} p !
$$

The theorem is proved.

The next statement follows directly from Theorem 1, (8) and (9).

Corollary 1: The following conditions are equivalent:

(a) $E A_{\infty}^{k} \leq c_{k}$ for every $k \geq 1$,

(b) $\quad E \sup _{t_{2}}\left(E\left(A_{\infty t_{2}}-A_{t} / \mathscr{F}_{t}\right)\right)^{k} \leq d_{k}$ for every $k \geq 1$.

Let $A_{t}$ be an integrable predictable increasing process. Denote

$$
\tau_{n}=\inf \left\{t_{1} \in R_{+}: A_{t_{1} t_{1}} \geq n\right\}
$$

and

$$
A_{t}^{n}=\int_{[0, t]} \Pi I_{\left[0, \tau_{n}\left[{ }^{2}\right.\right.}(s) d A_{s} .
$$

Note that $A_{t}^{n}$ is a predictable process and has all bounded moments. In fact, accord ing to [7], $A_{t}^{n}=\left(\int_{[0, t]} I_{\left[0, \tau_{n}\left[{ }^{2}\right.\right.}(s) d A_{s}\right)^{\pi}$, where we denote ()$^{\pi}$ the dual predictable projection [7] of the corresponding increasing predictable process. Further, again according to [7], for every $p \geq 1$ there exist constants $c_{p}$ such that

$$
E\left(A_{t}^{n}\right)^{p} \leq c_{p} E\left(\int_{[0, t]} I_{\left[0, \tau_{n}{ }^{2}{ }^{(s)} d A_{s}\right)}\right)^{p} \leq c_{p} n^{p} .
$$

Suppose now that $A_{t}$ and $B_{t}$ are two different increasing predictable processes; $A_{t}^{n}$ is defined by (10). Denote $\sigma_{n}=\inf \left\{t_{1} \in R_{+}: B_{t_{1} t_{1}} \geq n\right\}$ and

$$
B_{t}^{n}=\int_{[0, t]} \Pi I_{\left[0, \sigma_{n}{ }^{2}\right.}(s) d B_{s} .
$$

The next auxiliary result will be used for the proof of the main theorem.

Lemma 1: Let $A_{t}, B_{t}$ be two predictable increasing processes, $A_{t}^{n}$ is defined by (10), $B_{t}^{n}$ is defined by (11). Then

$$
\lim _{n \rightarrow \infty} E \int_{[0, t]} B_{s}^{n} d A_{s}^{n}=E \int_{[0, t]} B_{s} d A_{s} .
$$

Proof: Using (10), (11), and the definition of predictable projection [7], we can write

$$
\begin{gathered}
E \int_{[0, t]} B_{s}^{n} d A_{s}^{n}=E \int_{[0, t]}\left(\int_{[0, s]} \Pi_{\left[0, \sigma_{n}\left[{ }^{2}\right.\right.}(u) d B_{u}\right) \Pi_{\left[0, \tau_{n}\left[{ }^{2}\right.\right.}(s) d A_{s} \\
=E \int_{[0, t]}\left(\int_{[0, s]} \Pi_{\left[0, \sigma_{n}\left[{ }^{2}\right.\right.}(u) d B_{u}\right) I_{\left[0, \tau_{n}\left[{ }^{2}\right.\right.}(s) d A_{s} .
\end{gathered}
$$

Note that $\inf _{s \leq t} I_{\left[0, \tau_{n}\right]^{2}}(s) \rightarrow 1$ a.s. Thus, it is sufficient to prove that 


$$
\sup _{s \leq t}\left|B_{s}-\int_{[0, s]} \Pi I_{\left[0, \sigma_{n}\left[{ }^{2}\right.\right.}(u) d B_{u}\right|=\int_{[0, t]} \Pi I_{R^{2}}{ }^{2} \backslash\left[0, \sigma_{n}\left[{ }^{2}(s) d B_{s} \rightarrow 0\right. \text { a.s. }\right.
$$

The sequence $\left\{I_{R_{+}^{2} \backslash\left[0, \sigma_{n}\left[{ }^{2}\right.\right.}(s), n \geq 1\right\}$ is decreasing; hence, it is sufficient to prove that the last integral converges to zero in probability. But

$$
\begin{aligned}
& P\left\{\int_{[0, t]} \Pi I R_{+\backslash\left[0, \sigma_{n}{ }^{2}(s) d B_{s}>\varepsilon\right\}}\right\} \\
\leq & \frac{1}{\varepsilon} E \int_{[0, t]} I_{R_{+}^{2}}^{2} \backslash\left[0, \sigma_{n}\left[{ }^{2}(s) d B_{s} \rightarrow 0, n \rightarrow \infty,\right.\right.
\end{aligned}
$$

and we obtain the proof.

Further, we assume that $\sigma$-fields $\mathcal{F}_{t}^{1}$ and $\mathscr{F}_{t}^{2}$ are continuous on the right.

Lemma 2: Suppose $X_{t}$ is a bounded potential associated with increasing predictable process $A_{t}$, that has all finite moments, and $B_{t}$ is an increasing predictable process that has all moments. Then the following formula holds

$$
\begin{gathered}
E \int_{[0, \infty)} X_{t} d B_{t}+E \int_{[0, \infty)}\left(B_{t+-}+B_{t-+}-B_{t-}\right) d A_{t} \\
+E \int_{[0, \infty)} d_{1} X_{t} d_{2} B_{t}+E \int_{[0, \infty)} d_{1} B_{t} d_{2} X_{t}=0 .
\end{gathered}
$$

Proof: Denote $M_{t}^{1}=-E\left(A_{\infty t_{2}} / \mathcal{F}_{t}\right), M_{t}^{2}=-E\left(A_{t_{1} \infty} / \mathcal{F}_{t}\right), M_{t}=E\left(A_{\infty} / \mathcal{F}_{t}\right)$. Then $M_{t}^{1}$ is 1-martingale, $M_{t}^{2}$ is 2-martingale, $M_{t}$ is martingale, and from (1) $X_{t}=$ $M_{t}^{1}+M_{t}^{2}+M_{t}+A_{t}$. The process $B_{t}$ is 1-predictable, hence,

$$
E M_{t}^{1} B_{t}=E \int_{0}^{t_{1}} M_{s_{1}-} t_{2} d_{1} B_{s_{1} t_{2}}=E \lim _{\left|\lambda_{t}^{n}\right| \rightarrow 0} \sum_{i=0}^{n-1} M_{t_{1}^{i} t_{2}}^{1} \Delta_{t_{1}^{i} t_{2}}^{1} B \text { a.s. }
$$

where $M_{s_{1}{ }^{-} t_{2}}=M_{\left(s_{1}, t_{2}\right)-+}$, and $0=t_{1}^{0}<t_{1}^{1}<\ldots<t_{1}^{n}=t_{1}$ is the partition of $\left[0, t_{1}\right]$. We can write

$$
\sum_{i=0}^{n-1} M_{t_{1}^{i} t_{2}}^{1} \Delta_{t_{1}^{i} t_{2}}^{1} B=\sum_{i=0}^{n-1} \sum_{j=0}^{n-1} M_{i j}^{1} \square_{i j-1} B+\sum_{i=0}^{n-1} \sum_{j=0}^{n-1} \Delta_{i j-1}^{2} M^{1} \Delta_{i j-1}^{1} B .
$$

(Here and further we replace index $t_{1}^{i} t_{2}^{j}$ by index $i j$.) Under the assumption of continuity the $\sigma$-fields $\mathcal{F}_{t}^{1}$ and $\mathcal{F}_{t}^{2}$ on the right, the process $M_{t}^{1}$ satisfies the conditions of Theorems 3.4 and 4.2 [8]. Therefore, $M_{t}^{1}$ has a modification with limits in $Q_{t}^{i}, i=$ $2,3,4$ which is continuous in $Q_{t}^{1}$. For such modification

$$
\lim _{\left|\lambda_{t}^{n}\right| \rightarrow 0} \sum_{i=0}^{n-1} \sum_{j=0}^{n-1} M_{i j}^{1} \square_{i j-1} B=\int_{[0, t]} M_{s-+}^{1} d B_{s}
$$


Consequently, there exists

$$
\lim _{\left|\lambda_{t}^{n}\right| \rightarrow 0} \sum_{i=0}^{n-1} \sum_{j=0}^{n-1} \Delta_{i j-1}^{2} M^{1} \Delta_{i j-1}^{1} B=\int_{[0, t]} d_{1} B_{s} d_{2} M_{s}^{1}
$$

Furthermore, $\sum_{i=0}^{n-1} M_{t_{1}^{i} t_{2}}^{1} \Delta_{t_{1}^{i} t_{2}}^{1} B \leq B_{t_{t}} \sup E\left(A_{\infty} / \mathcal{F}_{t}\right)$; and the right-hand side of this inequality has all moments. Hence $\sum_{i=0}^{n-1} M_{t_{1}^{i} t_{2}}^{1} \Delta_{t_{1}^{i} t_{2}}^{1} B$ is uniformly integrable. Similarly, we can consider the sums $\sum_{i=0}^{n-1} \sum_{j=0}^{\substack{i=0 \\ n-1}} M_{i j}^{1} \square_{i j-1} B$ and obtain their uniform integrability. Thus, $\sum_{i=0}^{n-1} \sum_{j=0}^{n-1} \Delta_{i j-1}^{2} M^{1} \Delta_{i j-1}^{1} B$ is uniformly integrable and

$$
E M_{t}^{1} B_{t}=E \int_{[0, t]} M_{s-}^{1}+d B_{s}+E \int_{[0, t]} d_{1} B_{s} d_{2} M_{s}^{1}
$$

In the same way we obtain that

Also,

$$
E M_{t}^{2} B_{t}=E \int_{[0, t]} M_{s+-}^{2} d B_{s}+E \int_{[0, t]} d_{1} M_{s}^{2} d_{2} B_{s}
$$

$$
E M_{t} B_{t}=E \int_{[0, t]} M_{s} d B_{s}
$$

It is easy to check that the formula of integration by parts for two increasing processes $A_{t}$ and $B_{t}$ holds

$$
\begin{gathered}
E A_{t} B_{t}=E \int_{[0, t]} A_{s} d B_{s}+E \int_{[0, t]}\left(B_{s+-}+B_{s-+}-B_{s-}\right) d A_{s} \\
+E \int_{[0, t]} d_{1} A_{s} d_{2} B_{s}+E \int_{[0, t]} d_{1} B_{s} d_{2} A_{s} .
\end{gathered}
$$

Note that

$$
\begin{gathered}
E \int_{[0, t]}\left(M_{s}^{1}-M_{s-+}^{1}\right) d B_{s}=E \int_{[0, t]} \Pi^{1}\left(M_{s}^{1}-M_{s-+}^{1}\right) d B_{s} \\
=E \int_{[0, t]}\left(M_{s-+}^{1}-M_{s-+}^{1}\right) d B_{s}=0 .
\end{gathered}
$$

Therefore,

Similarly,

$$
E \int_{[0, t]} M_{s}^{1} d B_{s}=E \int_{[0, t]} M_{s-}^{1}+d B_{s}
$$




$$
E \int_{[0, t]} M_{s}^{2} d B_{s}=E \int_{[0, t]} M_{s+-}^{2} d B_{s}
$$

Adding (12)-(15) and using (16)-(17), we have

$$
\begin{gathered}
E X_{t} B_{t}=E \int_{[0, t]} X_{s} d B_{s}+E \int_{[0, t]}\left(B_{s+-}+B_{s-+}-B_{s-}\right) d A_{s} \\
\quad+E \int_{[0, t]} d_{1} X_{s} d_{2} B_{s}+E \int_{[0, t]} d_{1} B_{s} d_{2} X_{s}
\end{gathered}
$$

Letting $t \rightarrow \infty$ in the last equality and using the definition of the potential, we obtain the proof.

Consider the following sequence of predictable increasing processes

$$
B_{t}^{(0)}=1, B_{t}^{(1)}=A_{t}, B_{t}^{(2)}=\int_{[0, t]} B_{s}^{(1)} d A_{s}, \ldots, B_{t}^{(k)}=\int_{[0, t]} B_{s}^{(k-1)} d A_{s}, \ldots
$$

The next theorem is the main result of this section.

Theorem 4: Let $X_{t} \leq c$. Then for any $k \geq 1 E B_{\infty}^{(k)}<\infty$.

Proof: We use the induction in $k$.

(1) It is obvious that $E B_{\infty}^{(1)}=E A_{\infty}<\infty$.

(2) Suppose that $E B_{\infty}^{(k)}<\infty$ for some $k$, and prove that $E B_{\infty}^{(k+1)}<\infty$. Denote $\sigma_{n, k}=\inf \left\{t_{1} \in R_{+}: B_{t_{1} t_{1}}^{(k)} \geq n\right\}$ and

$$
B_{t}^{(k, n)}=\int_{[0, t]} \Pi I_{\left[0, \sigma{ }_{n, k}{ }^{2}\right.}(s) d B_{s}^{(k)}, k \geq 1, n \geq 1 .
$$

Let $A_{t}^{(n)}$ be defined by (10), $X_{t}^{(n)}$ be a potential associated with $A_{t}^{(n)}$. If we prove that under inductive hypothesis $E B_{\infty}^{(k+1, n)} \leq C<\infty$, then, letting $n \rightarrow \infty$ and using Lemma 1 , we obtain the proof.

Thus, prove that $E B_{\infty}^{(k+1, n)}<\infty$. For any process $B_{t}$ use the representation $B_{t}=B_{t-}+\Delta^{1} B_{t}+\Delta^{2} B_{t}-\square B_{t} \leq C$. Since

$$
\begin{gathered}
E B_{\infty}^{(k+1, n)}=E \int_{[0, \infty)} B_{t}^{(k, n)} d A_{t}^{(n)}=E \int_{[0, \infty)} B_{t}^{(k, n)} d A_{t}^{(n)} \\
+E \int_{[0, \infty)} \Delta^{1} B_{t}^{(k, n)} d A_{t}^{(n)}+E \int_{[0, \infty)} \Delta^{2} B_{t}^{(k, n)} d A_{t}^{(n)}-\int_{[0, \infty)} \square B_{t}^{(k, n)} d A_{t}^{(n)},
\end{gathered}
$$

it is sufficient to prove finiteness of each term in the right-hand side of the last equality.

(a) By Lemma 2 we can write

$$
E X_{\infty}^{(n)} B_{\infty}^{(k, n)}=E \int_{[0, \infty)} X_{t}^{(n)} d B_{t}^{(k, n)}
$$




$$
\begin{gathered}
+E \int_{[0, \infty)}\left(B_{t+-}^{(, n)}+B_{t-+}^{(k, n)}-B_{t-}^{(k, n)}\right) d A_{t}^{(n)} \\
+E \int_{[0, \infty)} d_{1} X_{t}^{(n)} d_{2} B_{t}^{(k, n)}+E \int_{[0, \infty)} d_{1} B_{t}^{(k, n)} d_{2} X_{t}^{(n)}
\end{gathered}
$$

where $A_{t}^{(n)}$ is defined by $(10)$, and $X_{t}^{(n)}$ is a potential associated with $A_{t}^{(n)}$. Taking (1) into account, we obtain that

$$
E \int_{[0, \infty)} d_{1} X_{t}^{(n)} d_{2} B_{t}^{(k, n)}=E \int_{[0, \infty)} d_{1}\left(A_{t}^{(n)}-A_{t_{1} \infty}^{(n)}\right) d_{2} B_{t}^{(k, n)}
$$

By the definition of the stochastic integral of the second kind

$$
\begin{gathered}
\int_{[0, \infty)} d_{1}\left(A_{t}^{(n)}-A_{t}^{(n)}\right) d_{2} B_{t}^{(k, n)}=\lim _{\left|\lambda_{t}^{n}\right| \rightarrow 0} \sum_{i, j=0}^{n-1} \Delta^{1}\left(A_{i \infty}^{(n)}-A_{i j}^{(n)}\right) \Delta_{i j}^{2} B^{(k, n)} \\
=\lim _{\left|\lambda_{t}^{n}\right| \rightarrow 0} \sum_{i, j=0}^{n-1} \sum_{l=j}^{n-1} \square_{i l} A^{(n)} \Delta_{i j}^{2} B^{(k, n)}=\lim _{\left|\lambda_{t}^{n}\right| \rightarrow 0} \sum_{i, l=0}^{n-1} B_{i l+1}^{(k, n)} \square_{i l} A^{(n)} \\
=\int B_{t-+}^{(k, n)} d A_{t}^{(n)}
\end{gathered}
$$

Hence,

Similarly,

$$
E \int_{[0, \infty)} d_{1} X_{t}^{(n)} d_{2} B_{t}^{(k, n)}=-E \int_{[0, \infty)} B_{t-+}^{(k, n)} d A_{t}^{(n)}
$$

Substituting (19) and (20) into (18), we obtain

$$
\begin{gathered}
E X_{\infty}^{(n)} B_{\infty}^{(k, n)}=E \int_{[0, \infty)} X_{t}^{(n)} d B_{t}^{(k, n)} \\
+E \int_{[0, \infty)}\left(B_{t+-}^{(k, n)}+B_{t-+}^{(k, n)}-B_{t-}^{(k, n)}\right) d A_{t}^{(n)}-E \int_{[0, \infty)} B_{t+-}^{(k, n)} d A_{t}^{(n)} \\
-E \int_{[0, \infty)} B_{t-+}^{(k, n)} d A_{t}^{(n)}=E \int_{[0, \infty)} X_{t}^{(n)} d B_{t}^{(k, n)}-E \int_{[0, \infty)} B_{t-}^{(k, n)} d A_{t}^{(n)}
\end{gathered}
$$

Since the left-hand side and the first term of the right-hand side of (21) are finite, we have by virtue of boundedness of $X$ and inductive hypothesis, that

$$
E \int_{[0, \infty)} B_{t-}^{(k, n)} d A_{t}^{(n)}<\infty
$$


(b) First, prove that

$$
E \int_{[0, \infty)} \square X_{t}^{(n)} d B_{t}^{(k, n)}=E \int_{[0, \infty)} \square A_{t}^{(n)} d B_{t}^{(k, n)} .
$$

Consider the following martingales

$$
M_{t}=X_{t}-A_{t}+E\left(A_{t_{1} \infty}-A_{\infty t_{2}} / \mathscr{F}_{t}\right)=E\left(A_{\infty} / \mathscr{F}_{t}\right)
$$

and

$$
M_{t}^{(n)}=X_{t}^{(n)}-A_{t}^{(n)}+E\left(A_{t_{1} \infty}^{(n)}-A_{\infty t_{2}}^{(n)} / \mathscr{F}_{t}\right)=E\left(A_{\infty}^{(n)} / \mathscr{F}_{t}\right)
$$

where $M_{\infty}=A_{\infty}$ and $M_{\infty}^{(n)}=A_{\infty}^{(n)}$. Therefore, from [4]

$$
\begin{gathered}
E A_{\infty}^{(n)} B_{\infty}^{(k, n)}=E \int_{[0, \infty)} E\left(A_{\infty}^{(n)} / \mathscr{F}_{t}\right) d B_{t}^{(k, n)} \\
=E \int_{[0, \infty)}\left(X_{t}^{(n)}-A_{t}^{(n)}+E\left(A_{t_{1} \infty}^{(n)}+A_{\infty t_{2}}^{(n)} / \mathscr{F}_{t}\right)\right) d B_{t}^{(k, n)} .
\end{gathered}
$$

Note that $M_{t}^{(n)}$ is a nonnegative measurable process, hence, by [7]

$$
\begin{aligned}
\Pi^{1} M_{t}^{(n)}= & E\left(M_{t}^{(n)} / \mathcal{F}_{t-}^{1}\right)=E\left(E\left(A_{\infty}^{(n)} / \mathcal{F}_{t}\right) / \mathcal{F}_{t-}^{1}\right) \\
& =E\left(A_{\infty}^{(n)} / \mathcal{F}_{t-+}\right)=M_{t-}^{(n)}+
\end{aligned}
$$

Similarly, $\Pi^{2} M_{t}^{(n)}=M_{t+-}^{(n)}$ and $\Pi M_{t}^{(n)}=M_{t-}^{(n)}$. From the definition of the predictable rejection we have

$$
\begin{aligned}
& E \int_{[0, \infty)} M_{t}^{(n)} d B_{t}^{(k, n)}=E \int_{[0, \infty)} M_{t-}^{(n)}+d B_{t}^{(k, n)} \\
= & E \int_{[0, \infty)} M_{t+-}^{(n)} d B_{t}^{(k, n)}=E \int_{[0, \infty)} M_{t-}^{(n)} d B_{t}^{(k, n)} .
\end{aligned}
$$

Thus, we can write $E A_{\infty}^{(n)} B_{\infty}^{(k, n)}$ in the four following ways:

$$
\begin{array}{r}
E A_{\infty}^{(n)} B_{\infty}^{(k, n)}=E \int_{[0, \infty)}\left(X_{t}^{(n)}-A_{t}^{(n)}+E\left(A_{t_{1} \infty}^{(n)}+A_{\infty t_{2}}^{(n)} / \mathscr{F}_{t}\right)\right) d B_{t}^{(k, n)}, \\
E A_{\infty}^{(n)} B_{\infty}^{(k, n)}=E \int_{[0, \infty)}\left(X_{t+-}^{(n)}-A_{t+-}^{(n)}+E\left(A_{t_{1} \infty}^{(n)}+A_{\infty t_{2}}^{(n)} / \mathscr{F}_{t+-}\right)\right) d B_{t}^{(k, n)}, \\
E A_{\infty}^{(n)} B_{\infty}^{(k, n)}=E \int_{[0, \infty)}\left(X_{t-}^{(n)}+-A_{t-}^{(n)}+E\left(A_{t_{1}}^{(n)}{ }_{\infty}+A_{\infty t_{2}}^{(n)} / \mathscr{F}_{t-+}\right)\right) d B_{t}^{(k, n)},
\end{array}
$$




$$
E A_{\infty}^{(n)} B_{\infty}^{(k, n)}=E \int_{[0, \infty)}\left(X_{t-}^{(n)}-A_{t-}^{(n)}+E\left(A_{t_{1}}^{(n)}{ }_{\infty}+A_{\infty t_{2}}^{(n)} / \mathscr{F}_{t-}\right)\right) d B_{t}^{(k, n)}
$$

Subtracting (24) and (25) from (23) and adding (26), we obtain, using predictability of $B_{t}^{(k, n)}$ and properties of conditional expectation, that

or

$$
E \int_{[0, \infty)}\left(\square X_{t}^{(n)}-\square A_{t}^{(n)}\right) d B_{t}^{(k, n)}=0
$$

$$
E \int_{[0, \infty)} \square X_{t}^{(n)} d B_{t}^{(k, n)}=E \int_{[0, \infty)} \square A_{t}^{(n)} d B_{t}^{(k, n)}
$$

The process $A_{t}^{(n)}$ admits the representation $A_{t}^{(n)}=A_{t}^{(n)^{c}}+A_{t}^{(n)^{c d}}+A_{t}^{(n)^{d c}}+A_{t}^{(n)^{d}}$, where $A_{t}^{(n)^{c}}$ is continuous, $A_{t}^{(n)^{c d}}$ is continuous in $t_{1}$ and purely discontinuous in $t_{2}$, $A_{t}^{(n)^{d c}}$ is purely discontinuous in $t_{1}$ and continuous in $t_{2}$, and, finally, $A_{t}^{(n)^{d}}$ is purely discontinuous. Therefore, only the integral $\underset{[0, \infty)}{E} \square B_{t}^{(k, n)} d A_{t}^{(n)^{d}}$ is nonzero, and

$$
\begin{gathered}
E \int_{[0, \infty)} \square B_{t}^{(k, n)} d A_{t}^{(n)}=\lim _{\left|\lambda_{t}^{n}\right| \rightarrow 0} E \sum_{i, j=0}^{n-1} \square_{i j} B^{(k, n)} \square_{i j} A^{(n)} \\
=E \int_{[0, \infty)} \square A_{t}^{(n)} d B_{t}^{(k, n)}
\end{gathered}
$$

From (27) and (28) we have

$$
E \int_{[0, \infty)} \square B_{t}^{(k, n)} d A_{t}^{(n)}=E \int_{[0, \infty)} \square X_{t}^{(n)} d B_{t}^{(k, n)}<4 c E B_{\infty}^{(k)}<\infty
$$

by the inductive hypothesis.

(c) Subtracting (25) from (23), we obtain

or

$$
E \int_{[0, \infty)}\left(\Delta^{1} X_{t}^{(n)}-\Delta^{1} A_{t}^{(n)}+\Delta^{1} A_{t_{1} \infty}^{(n)}\right) d B_{t}^{(k, n)}=0
$$

$$
E \int_{[0, \infty)} \Delta^{1} X_{t}^{(n)} d B_{t}^{(k, n)}=E \int_{[0, \infty)}\left(\Delta^{1} A_{t}^{(n)}-\Delta^{1} A_{t_{1} \infty}^{(n)}\right) d B_{t}^{(k, n)}
$$

Consider the integral in the right-hand side of (30)

$$
\begin{gathered}
\int_{[0, \infty)}\left(\Delta^{1} A_{t}^{(n)}-\Delta^{1} A_{t_{1} \infty}^{(n)}\right) d B_{t}^{(k, n)} \\
=\lim _{\left|\lambda_{t}^{n}\right| \rightarrow 0} \sum_{i, j=0}^{n} \Delta^{1}\left(A_{i j}^{(n)}-A_{i \infty}^{(n)}\right) \square_{i j} B^{(k, n)}
\end{gathered}
$$




$$
\begin{gathered}
=-\lim _{\left|\lambda_{t}^{n}\right| \rightarrow 0} \sum_{i, j=0}^{n-1} \sum_{l=j}^{n-1} \square_{i l} A^{(n)} \square_{i j} B^{(k, n)} \\
=-\lim _{\left|\lambda_{t}^{n}\right| \rightarrow 0} \sum_{i, l=0}^{n-1} \square_{i l} A^{(n)} \Delta_{i l+1}^{1} B^{(k, n)}=-\int_{[0, \infty)} \Delta^{1} B_{t}^{(k, n)} d A_{t}^{(n)} .
\end{gathered}
$$

From (30) and (31) we have

$$
E \int_{[0, \infty)} \Delta^{1} B_{t}^{(k, n)} d A_{t}^{(n)}=-E \int_{[0, \infty)} \Delta^{1} X_{t}^{(n)} d B_{t}^{(k, n)} .
$$

An absolute value of the right-hand side of the last equality does not exceed $2 c E B_{\infty}^{(k)}<\infty$, therefore

$$
E \int_{[0, \infty)} \Delta^{1} B_{t}^{(k, n)} d A_{t}^{(n)}<\infty
$$

(d) Similarly, using (24) instead of (25), one can prove that

Now, from $(a)-(d)$ we get

$$
E \int_{[0, \infty)} \Delta^{2} B_{t}^{(k, n)} d A_{t}^{(n)}<\infty
$$

$$
E B_{\infty}^{(k+1, n)}=E \int_{[0, \infty)} B_{t}^{(k, n)} d A_{t}^{(n)}<8 c E B_{\infty}^{(k)}<\infty .
$$

Letting $n \rightarrow \infty$ and using Lemma 1, we obtain the proof of the theorem.

In Theorem 4 we have proved that the boundedness of the potential implies the integrability of the process $B_{\infty}^{(k)} \leq A_{\infty}^{k}$.

\section{Application to the Local Time}

The process $\left\{Y_{t}, t \in R_{+}^{2}\right\}$ is said to have the local time $L_{t}(x)$ if $L_{t}(x)$ is an increasing process such that for all $t \in R_{+}^{2}$ and for all Borel locally integrable functions $f$ on $R$

$$
\int_{R} L_{t}(x) f(x) d x=\int_{[0, t]} f\left(Y_{s}\right) d s \quad \text { a.s. }
$$

A weak predictable set [4] $D(\omega) \subset R_{+}^{2}$ is called a stopping set if $[0, t] \subset D(\omega)$ when $t \in D(\omega)$, and the event $\{t \in D(\omega)\} \in \mathcal{F}_{t}$. We say that the process $Y$ belongs to a class $\&$ locally if there exists a sequence $\left\{D^{n}(\omega), n \geq 1\right\}$ of stopping sets and a sequence of processes $\left\{Y^{n}, n \geq 1\right\} \subset \&$ such that $D^{n} \subset D^{n+1}$ for every $n \geq 1, \bigcup_{n \geq 1} D^{n}=$ $R_{+}^{2}$, and $\left(Y_{t}-Y_{t}^{n}\right) I_{D^{n}}(t)=0$. Let $X_{t}$ be a local purely discontinuous strong martingale that has local characteristics $\left(a_{s}, \nu_{s}\right)$. This means that

- $\quad a_{s}$ and $\nu_{s}$ are $\mathcal{F}_{s}$-adapted,

- $\quad$ for each $s$ and $\omega \nu_{s}$ is a $\sigma$-finite measure on $R^{2} \backslash \Gamma$,

- for all Borel $B$ such that $\bar{B} \in R^{2} \backslash \Gamma$ is compact, $\sum_{s \leq t} I_{B}\left(\square X_{s}\right)-\int_{[0, t]} \nu_{s}(B) d s$ is 
a local strong martingale,

- $\quad X_{t}-\sum_{s \leq t} \square X_{s} I\left\{\left|\square X_{s}\right|>1\right\}-\int_{[0, t]} a_{s} d s$ is a local strong martingale, where the process $\int_{[0, t]} \nu_{s}(B) d s$ is a weak predictable projection [4] of the process $\sum_{s \leq t} I_{B}\left(\square X_{s}\right) . \quad X_{t}$ purely discontinuous means that $X_{t}$ is a uniform limit of

$$
\begin{gathered}
X_{t}^{\varepsilon}=\int_{[0, t]} a_{s} d s+\sum_{s \leq t} \square X_{s} I\left\{\left|\square X_{s}\right|>\varepsilon\right\} \\
-\int_{[0, t]} \int_{\varepsilon \leq|h| \leq 1} h \nu_{s}(d h) d s \text { as } \varepsilon \rightarrow 0 .
\end{gathered}
$$

If $1<\alpha<2$, let $\theta_{\alpha}(d h)=\xi_{\alpha}|h|^{-(1+\alpha)} d h$ be the Levy measure for a stable symmetric process $\left\{Z_{t}, t \in R_{+}^{2}\right\}$ of index $\alpha$, where $\xi_{\alpha}>0$ is the constant such that $\operatorname{Eexp}\left(i s Z_{t}\right)=\exp \left(-t_{1} t_{2}|s|^{\alpha}\right)$.

We assume that the following conditions hold:

(A) $\quad X_{t}$ is a local purely discontinuous strong martingale such that

(a) for some $K_{1} \sup _{s}\left|\int_{R} h^{2} \wedge 1 \nu_{s}(d h)\right| \leq K_{1}$ a.s.,

(b) for some $1<\alpha<2 \nu_{s}(d h)=\theta_{\alpha}(d h)$, if $|h|>1$,

(c) for some $1<\alpha<2, K_{2}>1$, any $0<\gamma<\frac{\alpha-1}{2}$

$$
\sup _{s} \int_{-1}^{1}|h|^{\alpha-\gamma}\left|\nu_{s}-\theta_{\alpha}\right|(d h) \leq K_{2} \text { a.s. }
$$

(d) $X_{t}$ does not have more than one jump along any line parallel to coordinate axes.

Example 3: Let $N_{t}, t \in R_{+}^{2}$ be a Poisson field with parameters $E N_{t}=E N_{t}^{2}=$ $\lambda t_{1} t_{2}$ (see, for example, [6]). Then $N_{t}-t_{1} t_{2}$ is a purely discontinuous local strong martingale with local characteristics $\nu_{s}(B)=I_{B}(1)$ and $a_{s}=1$. Consider local purely discontinuous strong martingale $\widetilde{N}_{t}$ with local characteristics $\widetilde{\nu}_{s}(B)=I_{B}(1)+\theta_{\alpha}(B)$ for all Borel $B$ such that $\widetilde{B} \in R^{2} \backslash \Gamma$ is compact, where $\theta_{\alpha}(d h)$ is the Levy measure described above. Then $\widetilde{N}_{t}$ satisfies conditions $(A)$.

Let $Q_{t}(\omega, \cdot)$ be a regular conditional probability distribution for $\mathcal{F}_{t}$. That is for every $A \in \mathcal{F} Q_{t}(\cdot, A)$ is $\mathcal{F}_{t}$-measurable, for every $\omega Q_{t}(\omega, \cdot)$ is a probability measure on $\mathscr{F}$, and $Q_{t}(\cdot, A)=P\left(A / \mathcal{F}_{t}\right)$ a.s. for every $A \in \mathcal{F} . Q_{t}$ exists since $\mathcal{F}_{t}$ is the completion of a countably generated $\sigma$-fields and $X_{t}$ is real-valued. Denote $Q_{t} Y(\omega)=$ $\int Y\left(\omega^{\prime}\right) Q_{t}\left(\omega, d \omega^{\prime}\right)=E\left(Y(\omega) / \mathcal{F}_{t}\right)$.

Fix $t_{0} \in R_{+}^{2}, \lambda>0$. It was proved in [9] that there exists a nonnegative Borel in $x$ bounded function $V_{t_{0}}(\lambda, x)(\omega)$ such that for every Borel $A \in R$

$$
Q_{t_{0}}\left(\int_{[0, \infty)} e^{-\lambda t_{1}-\lambda t_{2}} I_{A}\left(X_{t+t_{0}}\right) d t\right)(\omega)=\int_{A} V_{t_{0}}(\lambda, x)(\omega) d x
$$

Further, the process $\left\{U_{t}(\lambda, x)=e^{-\lambda t_{1}-\lambda t_{2}} V_{t}(\lambda, x), \mathscr{F}_{t}, t \in Q_{+}^{2}\right\}$ for a.e. $x$ is a weak submartingale, supermartingale, and a.s. for any $s \in Q_{+}^{2}$ and for any sequence $\left\{t_{n}, n \geq 1\right\} \subset Q^{2}+$ such that $t_{n} \downarrow s, n \rightarrow \infty$, for a.e. $x E U_{t_{n}}(\lambda, x) \rightarrow E U_{s}(\lambda, x), n \rightarrow \infty$. Here we denote $Q_{+}^{2}=Q_{+} \times Q_{+}, Q_{+} Q \cap[0, \infty)$. 
Under the assumption

(B) for a.e. $x$ the process $\left(U_{t}(\lambda, x), \mathscr{F}_{t}, t \in R_{+}^{2}\right)$ is a weak submartingale, supermartingale and for every $s \in R_{+}^{2} \quad E U_{t}(\lambda, x) \rightarrow E U_{s}(\lambda, x)$ if $t \rightarrow s, t>s$, $t \in R_{+}^{2}$.

The process $\stackrel{+}{U}_{t}(\lambda, x)$ for a.e. $x$ allows the representation [9]

$$
U_{t}(\lambda, x)=M_{t}(\lambda, x)+L_{t}(\lambda, x)
$$

where $M_{t}(\lambda, x)$ is a weak martingale, and $L_{t}(\lambda, x)$ is an increasing predictable process. Note that $U_{t}(\lambda, x)$ is a bounded potential associated with $L_{t}(\lambda, x)$.

Fix $\lambda>0$ and let

$$
L_{t}(x)=\int_{[0, t]} e^{\lambda s_{1}+\lambda s_{2}} d L_{s}(\lambda, x) .
$$

Then from [9] $L_{t}(x)$ is a local time for $X_{t}$, and it does not depend on $\lambda$. Consider the sequences of increasing predictable processes

$$
\begin{gathered}
C_{t}^{(0)}(\lambda, x)=1, C_{t}^{(1)}(\lambda, x)=L_{t}(\lambda, x), C_{t}^{(2)}(\lambda, x)=\int_{[0, t]} C_{s}^{(1)}(\lambda, x) d L_{s}(\lambda, x), \\
\ldots, C_{t}^{(k)}(\lambda, x)=\int_{[0, t]} C_{s}^{(k-1)}(\lambda, x) d L_{s}(\lambda, x), \ldots
\end{gathered}
$$

and

$$
\begin{gathered}
C_{t}^{(0)}(x)=1, C_{t}^{(1)}(x)=L_{t}(x), C_{t}^{(2)}(x)=\int_{[0, t]} C_{s}^{(1)}(x) d L_{s}(x), \ldots, \\
C_{t}^{(k)}(x)=\int_{[0, t]} C_{s}^{(k-1)}(x) d L_{s}(x), \ldots
\end{gathered}
$$

Since potential $U_{t}(\lambda, x)$ is bounded, from Theorem 4 for any $k \geq 1 E C_{\infty}^{(k)}(\lambda, x)<\infty$. Therefore, using $(35)$, we have that $E C_{t}^{(k)}(x)<\infty$.

Suppose that one more condition holds:

$(C) \quad E \sup _{t_{2}}\left(E\left(L_{\infty t_{2}}(\lambda, x)-L_{t}(\lambda, x) / \mathscr{F}_{t}\right)\right)^{k} \leq d_{k}$ for every $k \geq 1$.

Then, from Corollary $1 E\left[L_{\infty}(\lambda, x)\right]^{k}<\infty$ for every $k \geq 1$. Hence, $E\left[L_{t}(x)\right]^{k} \leq$ $e^{k \lambda\left(t_{1}+t_{2}\right)} E\left[L_{t}(\lambda, x)\right]^{k}<\infty, k \geq 1$.

Now we can formulate the result:

Theorem 5: Suppose $X_{t}$ is a purely discontinuous local strong martingale and $X_{t}$ satisfies conditions $(A)-(B)$. Then

(a) there exists jointly measurable continuous in $Q_{t}^{1}$ increasing process $L_{t}(x)$ such that (34) holds,

(b) for any $k \geq 1 E C_{t}^{(k)}(x)<\infty$,

(c) if, in addition, condition $(C)$ holds, then for every $t \in R_{+}^{2}$ and for every $x$ $L_{t}(x)$ has moments of all orders.

Remark 3: The existence and estimations of moments of local time for oneparameter purely discontinuous local martingales were obtained in [1]. 


\section{Acknowledgement}

The authors thank the associate editor, Professor A. Rosalsky, and the anonymous referee for their valuable suggestions.

\section{References}

[1] Bass, R.F., Local times for a class of purely discontinuous martingales, $Z$. Wahrsch. verw. Gebiete 67 (1984), 433-459.

[2] Cairoli, R. and Walsh, J.B., Stochastic integrals in the plane, Acta Mathematica 134:1-2 (1975), 111-183.

[3] Dellacherie, C. and Meyer, P.A., Probabilités et Potentiel, Hermann, Paris 1980.

[4] Gushchin, A.A., On the general theory of random fields on the plane, Russian Math. Surveys 37:6 (1982), 55-80.

[5] Mazziotto, G. and Merzbach, E., Regularity and decomposition of two-parameter supermartingales, J. Multiv. Anal. 17 (1985), 38-55.

[6] Mazziotto, G. and Szpirglas, J., Equations du filtrage pour un processus de Poisson mélangé à deux indices, Stochastics 4 (1980), 89-119.

[7] Merzbach, E. and Zakai, M., Predictable and dual predictable projections of two-parameter stochastic processes, Z. Wahrsch. verw. Gebiete 53 (1980), 263269.

[8] Millet, A. and Sucheston, L, On regularity of multiparameter amarts and martingales, Z. Wahrsch. verw. Gebiete 56 (1981), 21-45.

[9] Mishura, Yu.S. and Oltsik, Ya.A., Potentials and local times associated with two-parameter purely discontinuous strong martingales, Prob. Theory and Math. Statistics 56 (1997), 73-86 (in Ukrainian). 


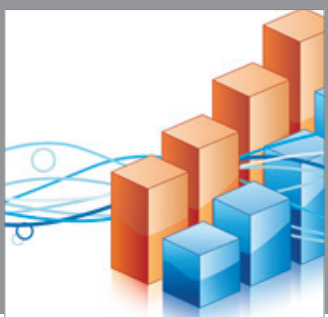

Advances in

Operations Research

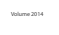

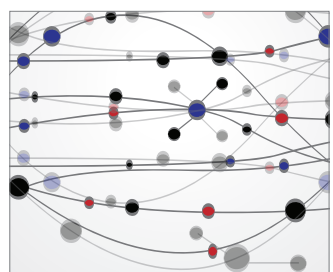

\section{The Scientific} World Journal
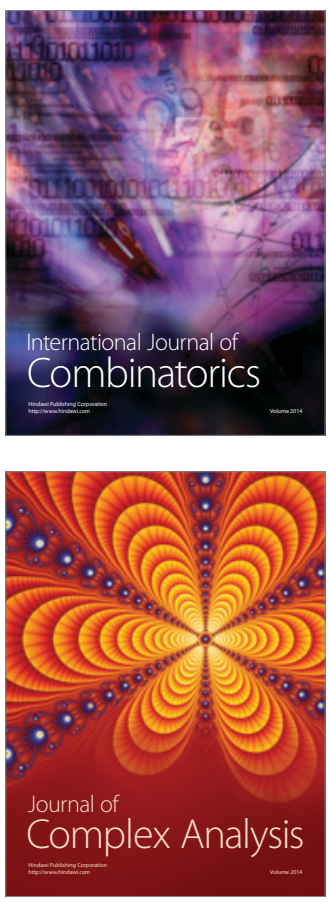

International Journal of

Mathematics and

Mathematical

Sciences
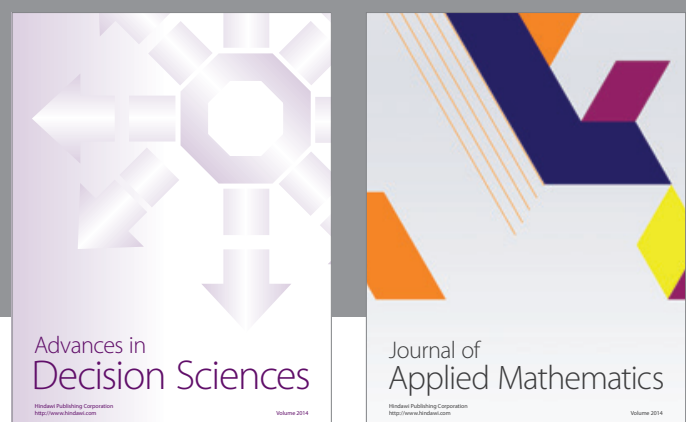

Journal of

Applied Mathematics
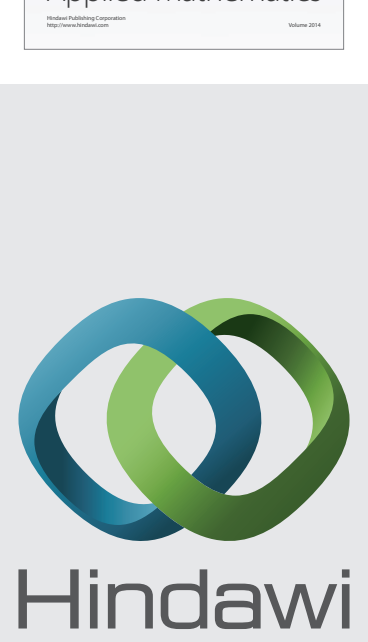

Submit your manuscripts at http://www.hindawi.com
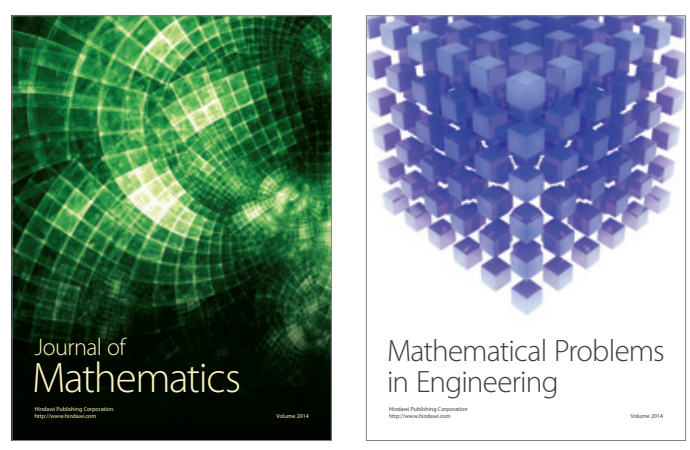

Mathematical Problems in Engineering
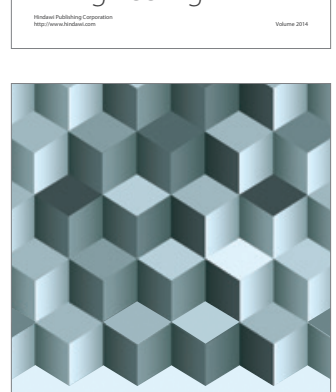

Journal of

Function Spaces
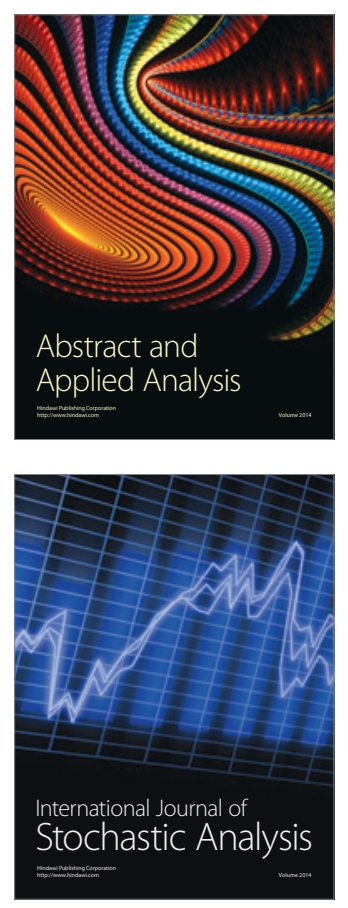

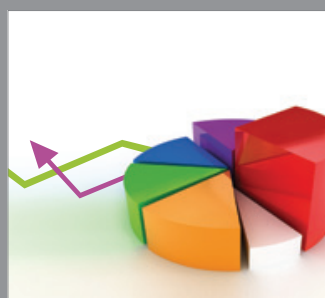

ournal of

Probability and Statistics

Promensencen
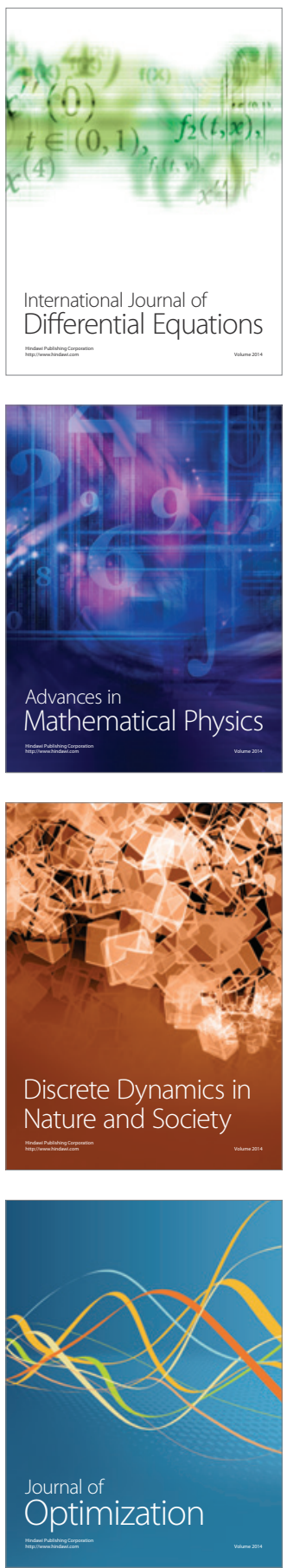